\title{
Limited-sampling strategy models for estimating the pharmacokinetic parameters of 4-methylaminoantipyrine, an active metabolite of dipyrone
}

\section{G. Suarez-Kurtz ${ }^{1,2}$, F.M. Ribeiro ${ }^{1}$, R.C.E. Estrela ${ }^{1}$, F.L. Vicente ${ }^{1,2}$ and C.J. Struchiner ${ }^{1}$}

\author{
1Divisão de Farmacologia, Coordenação de Pesquisa, \\ Instituto Nacional de Câncer, Rio de Janeiro, RJ, Brasil \\ 2Unidade de Farmacologia Clínica, Santa Casa da Misericórdia, \\ Rio de Janeiro, RJ, Brasil
}

\section{Correspondence \\ G. Suarez-Kurtz \\ Instituto Nacional de Câncer, CPQ \\ Praça da Cruz Vermelha, 23 \\ 20130-230 Rio de Janeiro, RJ \\ Brasil \\ Fax: +55-21-506-6376 \\ E-mail: kurtz@ inca.org.br \\ Research supported by CNPq, Fundação Ary Frauzino (FAF) and FAPERJ. G. Suarez-Kurtz and C.J. Struchiner are Senior Investigators of $\mathrm{CN} \mathrm{Pq}$. \\ R.C.E. Estrela and F.M. Ribeiro were recipients of fellowships from CN Pq/PIM ED.}

Received March 8, 2001 Accepted August 21, 2001

\section{Abstract}

Bioanalytical data from a bioequivalence study were used to develop limited-sampling strategy (LSS) models for estimating the area under the plasma concentration versus time curve (AUC) and the peak plasma concentration $\left(\mathrm{C}_{\max }\right)$ of 4-methylaminoantipyrine (MAA), an active metabolite of dipyrone. Twelve healthy adult male volunteers received single $600 \mathrm{mg}$ oral doses of dipyrone in two formulations at a 7-day interval in a randomized, crossover protocol. Plasma concentrations of MAA $(\mathrm{N}=336)$, measured by HPLC, were used to develop LSS models. Linear regression analysis and a "jack-knife" validation procedure revealed that the $\mathrm{AUC}_{0-\infty}$ and the $\mathrm{C}_{\max }$ of MAA can be accurately predicted $\left(\mathrm{R}^{2}>0.95\right.$, bias $<1.5 \%$, precision between 3.1 and $8.3 \%$ ) by LSS models based on two sampling times. Validation tests indicate that the most informative 2-point LSS models developed for one formulation provide good estimates $\left(\mathrm{R}^{2}>0.85\right)$ of the $\mathrm{AUC}_{0-\infty}$ or $\mathrm{C}_{\max }$ for the other formulation. LSS models based on three sampling points $\left(1.5,4\right.$ and $24 \mathrm{~h}$ ), but using different coefficients for $\mathrm{AUC}_{0-\infty}$ and $\mathrm{C}_{\max }$, predicted the individual values of both parameters for the enrolled volunteers $\left(R^{2}>0.88\right.$, bias $=-0.65$ and $-0.37 \%$, precision $=4.3$ and $7.4 \%$ ) as well as for plasma concentration data sets generated by simulation $\left(\mathrm{R}^{2}>0.88\right.$, bias $=-1.9$ and $8.5 \%$, precision $=5.2$ and $\left.8.7 \%\right)$. Bioequivalence assessment of the dipyrone formulations based on the $90 \%$ confidence interval of log-transformed $\mathrm{AUC}_{0-\infty}$ and $\mathrm{C}_{\max }$ provided similar results when either the best-estimated or the LSS-derived metrics were used.

\section{Introduction}

Dipyrone (noramidopyrine methanesulfonate, metamizole) is an effective analgesic, antipyretic and anti-inflammatory drug that has been widely used since its introduction in 1922. Dipyrone is a typical prodrug,
Key words

- Dipyrone

- 4-M ethylaminoantipyrine

- Limited-sampling models

- Pharmacokinetics

- Bioequivalence and its pharmacokinetics has been extensively investigated (1). After oral administration, dipyrone is non-enzymatically hydrolyzed in the gastrointestinal tract to 4methylaminoantipyrine (MAA), which is rapidly and nearly completely absorbed, reaching peak levels within 1-2 h. MAA is further 
metabolized to 4-formylaminoantipyrine, and 4-aminoantipyrine, the latter being acetylated to 4-acetylaminoantipyrine (2-4). Dipyrone is present in more than one hundred pharmaceutical products marketed in Brazil, the vast majority of which was not subjected to bioavailability studies prior to registration. Recently, however, we performed a bioequivalence study of two different formulations of dipyrone in association with caffeine and isometheptene produced by the same manufacturer (5). Because the analgesic effect of dipyrone correlates with the time course of MAA concentrations in serum (6), pharmacokinetic parameters pertaining to this active metabolite are appropriate for assessing the bioequivalence of dipyrone formulations. In the present study, the plasma concentrations of MAA were used to develop limited-sampling strategy (LSS) models to estimate both the area under the plasma concentration versus time curve (AUC) and the peak plasma concentration $\left(\mathrm{C}_{\max }\right)$ of MAA. Strategies using a limited number of samples and proven to be sufficiently robust to allow accurate estimation of individual pharmacokinetic parameters could be very valuable for bioequivalence studies, with reduced costs of sample acquisition and analysis, reduced study duration and avoidance of sampling at "unsociable" hours $(7,8)$.

\section{Material and Methods}

\section{Clinical protocol}

This open label, randomized study used a standard two-sequence, two-period crossover design in which the treatment phases were separated by a 7-day washout interval. The study protocol was approved by the Ethics Committee of Santa Casa da Misericórdia, Rio de Janeiro, and all participants provided written informed consent. Twelve healthy adult male volunteers aged 19 to 26 years (mean \pm SD: $22.1 \pm 2.3$ years) and weighing $55-72 \mathrm{~kg}(64.1 \pm 5.8 \mathrm{~kg})$ completed the study. These volunteers were non-smokers and had no clinically significant abnormalities, as determined 2 weeks before the start of the study, based on medical history, physical examination, electrocardiogram, and standard laboratory test results (blood cell count, biochemical profile and urinalysis). The enrolled volunteers had not used any investigational drug during the 6 months preceding the present study. Prescription drugs were not allowed during the study.

In each treatment phase, the volunteers arrived at the Clinical Pharmacology Unit at 7:00 pm. After an overnight (>10 h) fast, a catheter was introduced into a superficial vein, and a baseline (pre-dosing) blood sample was collected. Each volunteer then received $600 \mathrm{mg}$ dipyrone, as two 300-mg tablets of one of the two formulations (A, B) under study, with $200 \mathrm{ml}$ water. Six volunteers received the two formulations in one sequence, and the other six in the opposite sequence, in a balanced crossover design. Two hours after drug administration, the volunteers received a standard breakfast consisting of $200 \mathrm{ml}$ homogenized milk, $200 \mathrm{ml}$ orange juice, two slices of bread with ham and cheese, and one apple. Five, 8 and $12 \mathrm{~h}$ after drug administration, a standard lunch, snack and dinner were served. The volunteers remained at the Clinical Pharmacology Unit until collection of the 24-h blood sample and returned to the unit $48 \mathrm{~h}$ after drug administration.

Eight-milliliter blood samples were drawn into heparinized tubes 5-10 min before (zero time), and 0.33, 0.66, 1, 1.5, 2, 3, 4, 5, 6, 8, 10,24 and $48 \mathrm{~h}$ after administration of dipyrone. The blood samples were centrifuged within $30 \mathrm{~min}$ after collection, and the plasma was separated and stored at $-20^{\circ} \mathrm{C}$. The determination of the plasma concentrations of MAA was performed by HPLC using aminophylline $(10 \mu \mathrm{g} / \mathrm{ml})$ as internal standard. In this method, the internal standard was added to $1 \mathrm{ml}$ plasma, and after 
vortex mixing for $10 \mathrm{~s}, 50 \mu \mathrm{l}$ trichloroacetic acid $(30 \%)$ was added. The sample was vortex mixed again and centrifuged at $11,000 \mathrm{~g}$ for $10 \mathrm{~min}$. The supernatant $(100 \mu \mathrm{l})$ was then applied directly to the HPLC reversed phase column ODS-23 (100 x $4.6 \mathrm{~mm}$, Waters Spherisorb). The column was maintained at ambient temperature and eluted with a gradient of aqueous trifluoracetic acid (TFA) $(0.15 \%, \mathrm{v} / \mathrm{v})$ and $80 \%$ acetonitrile-TFA $(0.05 \%, \mathrm{v} / \mathrm{v})$ over a period of $15 \mathrm{~min}$. The column was then allowed to re-equilibrate with TFA for 3 min before the next injection. This method has a quantification limit of 0.1 $\mu \mathrm{g} / \mathrm{ml}$ for MAA. Standard curves were linear in the evaluated concentration range (0.1-30 $\left.\mu \mathrm{g} / \mathrm{ml}, \mathrm{R}^{2}=0.98\right)$ and the overall precision, as evaluated by the coefficients of variation obtained from independently prepared control plasma samples $(0.5-5.0 \mu \mathrm{g} / \mathrm{ml})$, ranged from $5.6 \%$ (intraday, $\mathrm{N}=6$ ) to $16.2 \%$ (interday, $\mathrm{N}=8$ ).

\section{Drugs}

Formulation A was commercially available as Neosaldina ${ }^{\circledR}$ (batch 9811341, Knoll Produtos Químicos e Farmacêuticos Ltda., Rio de Janeiro, RJ, Brazil), while formulation $\mathrm{B}$ was a novel formulation under development by the same manufacturer (batch 9811368). Both formulations contained 300 $\mathrm{mg}$ dipyrone, $30 \mathrm{mg}$ caffeine base and $50 \mathrm{mg}$ isometheptene hydrochloride.

\section{Pharmacokinetic analysis}

The value of $\mathrm{C}_{\max }$ and the time to reach it were determined from the individual plasma drug data. A non-compartmental model for extravascular input, provided by the WinNonlin Professional 3.1 software (9) was used for calculation of the pharmacokinetic parameters $\mathrm{k}_{\mathrm{el}}$ (terminal elimination rate constant), $\mathrm{t}_{1 / 2}$ (terminal half-life), $\mathrm{AUC}_{0-48}$ (area under the plasma drug concentration versus time curve between 0 to $48 \mathrm{~h}$ ), and the ex- trapolated $\mathrm{AUC}_{0-\infty}$ (AUC from 0 to infinity). The AUC thus obtained are taken as the "best estimates" of parameter values (see below).

\section{LSS development}

All-subset linear regression analysis (10) of the $\mathrm{AUC}_{0-\infty}$ or $\mathrm{C}_{\max }$ best estimates against $\mathrm{C}_{\text {time }}$ (independent variables) was carried out in order to develop LSS equations to estimate the $\mathrm{AUC}_{0-\infty}$ or $\mathrm{C}_{\max }$ for MAA following administration of each dipyrone formulation. Computations were carried out using function leaps (11) in Splus 4.0 (12). This analysis produced equations of the form $\mathrm{AUC}_{0-\infty}$ or $\mathrm{C}_{\max }=\mathrm{A}_{0}+\mathrm{A}_{1} \times \mathrm{C}_{1}+\mathrm{A}_{2} \times \mathrm{C}_{2} \ldots$ $A_{n} \times C_{n}$, where $A_{n}$ are coefficients and there is a variable number $n$ of samples. Regression equations were then ranked according to the $\mathrm{R}^{2}$ criterion in order to identify those that provided the best fit for 1 to 10 timed plasma samples. The LSS-derived $\mathrm{AUC}_{0-\infty}$ or $\mathrm{C}_{\max }$ estimates were then compared with the best estimates of these parameters for the data sets of each volunteer. The bias of these LSS-derived estimates was assessed by calculating the mean percentage of difference (MD\%) from the best estimates, where MD\% $=[($ derived estimate - best estimate $) /$ best estimate] $\mathrm{x} 100 \%$, and precision was assessed by calculating the mean absolute percentage of difference (MAD\%), where $\mathrm{MAD} \%=[($ derived estimate - best estimate/ best estimate] x $100 \%$.

The LSS models developed in the current study were validated by three procedures. The first is the jack-knife prediction (13), which is made when the regression equations to estimate $\mathrm{AUC}_{0-\infty}$ or $\mathrm{C}_{\max }$ are derived using the $n$ fixed concentrations of choice from 11 of the volunteers, and these equations are used to predict the $\mathrm{AUC}_{0-\infty}$ or the $\mathrm{C}_{\max }$ of the 12th volunteer, respectively. Thus, for each subset of sample times, slightly different regression equations are used to predict the $\mathrm{AUC}_{0-\infty}$ or the $\mathrm{C}_{\max }$ for each 
volunteer.

As a second validation approach (7), the 12 plasma concentration data sets for formulation B (training set) were used to estimate the regression coefficients in 2-point LSS models. These coefficients and the concentrations observed at the same respective times, but after administration of formulation A (validation set) were used to estimate the individual $\mathrm{AUC}_{0-\infty}$ or $\mathrm{C}_{\max }$ for the latter formulation. The $\mathrm{AUC}_{0-\infty}$ and $\mathrm{C}_{\max }$ thus obtained were then compared to the best estimates available for either parameter in each of the 12 volunteers.

As a third validation approach, we simulated new data as follows. Starting with the average plasma concentration data points obtained from a published study (14), in which 15 healthy male volunteers were given single oral doses $(750 \mathrm{mg})$ of dipyrone, we used the ADAPT II software (14) to fit a twocompartment model, allowing for a lag time (model 2lagk) to the data. At the end of this step, we obtained a set of six descriptive parameters for the average plasma concentration versus time curve. Next, we used the

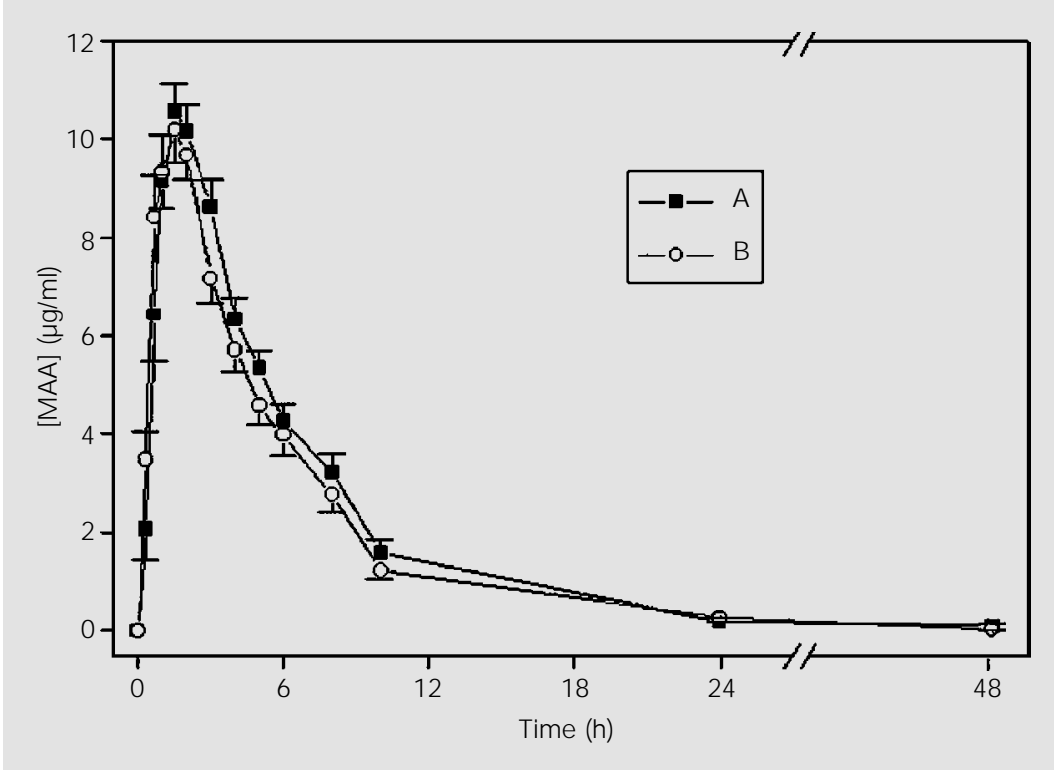

Figure 1. Plasma 4-methylaminoantipyrine (MAA) concentration-time curves of Brazilian healthy volunteers after single oral doses $(600 \mathrm{mg})$ of two dipyrone formulations (A, B). Data are reported as means \pm SEM.
SIM module of ADAPT II (option 4, population estimates with output noise) to generate 24 sets of plasma concentration data points at the same sampling times as used in the current study. The individual values of $\mathrm{C}_{\max }$ and $\mathrm{AUC}_{0-\infty}$ for each simulated set were calculated as described above for the volunteer data sets and represent the best estimates for these metrics. These values were then compared with the corresponding LSS-derived metrics for the same simulated data sets.

\section{Bioequivalence analysis}

The $90 \%$ confidence intervals of the individual ratio (formulation $\mathrm{A} /$ formulation $\mathrm{B}$ ) of the log-transformed values of the bestestimated $\mathrm{AUC}_{0-\infty}$ and $\mathrm{C}_{\max }$ were used to assess the bioequivalence of the two dipyrone formulations administered to the volunteers (15). The same procedure was applied to the 3-point LSS-derived $\mathrm{AUC}_{0-\infty}$ and $\mathrm{C}_{\max }$ to explore the usefulness of the LSS approach in bioequivalence studies.

\section{Statistical analysis}

The specific statistical tests applied to the data sets are indicated in the text. The level of significance was set at $\mathrm{P}<0.05$.

\section{Results}

Both dipyrone formulations were well tolerated by the enrolled volunteers, with no adverse effects being reported.

\section{Pharmacokinetic data}

The plasma MAA concentration-time curves for both dipyrone formulations are shown in Figure 1, and the pharmacokinetic parameters derived from these curves are summarized in Table 1. The data reveal large interindividual variability (coefficients of variation $>30 \%$ ) in several parameters, i.e., $\mathrm{AUC}_{0-48}, \mathrm{AUC}_{0-\infty}, \mathrm{t}_{1 / 2}$ and $\mathrm{k}_{\mathrm{el}}$, but no signifi- 
cant differences between the mean values of these parameters for each dipyrone formulation (Mann-Whitney rank sum test).

\section{Limited-sampling models for $\mathrm{AUC}_{0-\infty}$}

The plasma concentration data sets and an all-subset regression approach were used to identify the most informative sampling times, using 1 to 10 samples to estimate the $\mathrm{AUC}_{0-\infty}$ of MAA for each formulation tested. The results of this analysis (Table 2) indicate that the most informative strategies depend on the dipyrone formulation; in the case of two samples, the most accurate estimates of AUC $_{0-\infty}$ were obtained from data points at 24 $\mathrm{h}$ and at $5 \mathrm{~h}$ (formulation $\mathrm{A}$ ) or $4 \mathrm{~h}$ (formulation B). The corresponding equations are:

Formulation A:

$\mathrm{AUC}_{0-\infty}=7.87+9.71 * \mathrm{C}_{5}+50.15 * \mathrm{C}_{24}$

Formulation B:

$\mathrm{AUC}_{0-\infty}=-3.47+10.48 * \mathrm{C}_{4}+27.64 * \mathrm{C}_{24}$

These equations provided accurate estimates $\left(\mathrm{R}^{2}>0.96\right.$, bias $<1.5 \%$, precision $=5.4$ and $8.3 \%$; Table 2) of the $\mathrm{AUC}_{0-\infty}$ for each formulation. Increasing the number of sampling points to more than two increases $\mathrm{R}^{2}$ by $<4 \%$ and adds little to the bias and the precision of the estimates of $\mathrm{AUC}_{0-\infty}$, as compared to the respective values for 2-point sampling for each formulation (Table 2).

Diagnostic jack-knife plots (Methods) of the best-estimated $\mathrm{AUC}_{0-\infty}$ versus the LSSderived $\mathrm{AUC}_{0-\infty}$ for either formulation (Figure $2 A, B)$ show agreement $\left(R^{2}>0.90\right.$, bias $<3.5 \%$, precision $<11.9 \%$ ) between observed and predicted quantities. Residual plots (data not shown) indicate no need to search for either additional variable transformations or non-linear relationships for either data set. Figure $2 \mathrm{C}$ shows another validation approach of the 2-point LSS models, in which the most informative LSS equation developed for formulation B and the concentrations observed at the same respective times, but after administration of formulation $\mathrm{A}$, were used to estimate the individual $\mathrm{AUC}_{0-\infty}$ for the latter formulation (Methods). The $\mathrm{AUC}_{0-\infty}$ thus obtained showed good correlation $\left(\mathrm{R}^{2}=0.85\right.$, $\mathrm{P}<0.001)$ with the best-estimated parameter values.

The most-informative 2-point LSS models (Table 2) include the 24-h data points, which occur at a late time in the elimination phase of the plasma concentration versus time curve (Figure 1), when the MAA concentrations are close to or below the quantification limit of the analytical method used. To circumvent this limitation, LSS equations derived for two sampling points, but

\begin{tabular}{|c|c|c|}
\hline \multirow[t]{2}{*}{ Parameter } & \multicolumn{2}{|c|}{ Dipyrone formulation } \\
\hline & A & B \\
\hline \multicolumn{3}{|l|}{$C_{\max }(\mu \mathrm{g} / \mathrm{ml})$} \\
\hline Mean \pm SD & $11.0 \pm 1.81$ & $10.6 \pm 2.1$ \\
\hline Geometric mean & 10.8 & 10.4 \\
\hline \multicolumn{3}{|l|}{$\mathrm{AUC}_{0-48}\left(\mu \mathrm{g} \mathrm{h} \mathrm{ml}^{-1}\right)$} \\
\hline Mean $\pm S D$ & $64.2 \pm 25.8$ & $60.0 \pm 18.5$ \\
\hline Geometric mean & 60.1 & 56.9 \\
\hline \multicolumn{3}{|l|}{$\operatorname{AUC}_{0_{-\infty}}\left(\left(\mu \mathrm{g} \mathrm{h} \mathrm{ml}^{-1}\right)\right.$} \\
\hline Mean \pm SD & $68.3 \pm 26.8$ & $63.7 \pm 18.9$ \\
\hline Geometric mean & 64.1 & 60.5 \\
\hline \multicolumn{3}{|l|}{$\mathrm{C}_{\max } / \mathrm{AUC}_{0-48}\left(\mathrm{~h}^{-1}\right)$} \\
\hline Mean \pm SD & $0.17 \pm 0.04$ & $0.18 \pm 0.05$ \\
\hline Geometric mean & 0.17 & 0.17 \\
\hline \multicolumn{3}{|l|}{$\mathrm{Kel}_{\mathrm{el}}\left(\mathrm{h}^{-1}\right)$} \\
\hline Mean \pm SD & $0.24 \pm 0.11$ & $0.22 \pm 0.12$ \\
\hline Geometric mean & 0.22 & 0.19 \\
\hline \multicolumn{3}{|l|}{$\mathrm{t}_{1 / 2}(\mathrm{~h})$} \\
\hline Mean \pm SD & $3.4 \pm 1.5$ & $4.4 \pm 2.8$ \\
\hline Geometric mean & 3.1 & 3.7 \\
\hline \multicolumn{3}{|l|}{$\mathrm{T}_{\max }(\mathrm{h})$} \\
\hline Median & 1.6 & 1.5 \\
\hline Range & $1-2$ & $0.66-2$ \\
\hline
\end{tabular}

aEach volunteer $(\mathrm{N}=12)$ ingested $600 \mathrm{mg}$ of either dipyrone formulation at a 7-day interval. $\mathrm{C}_{\max }=$ peak plasma concentration; $\mathrm{AUC}=$ area under the plasma drug concentration versus time curve between 0 to $48 \mathrm{~h}\left(\mathrm{AUC}_{0-48}\right)$ and from 0 to infinity $\left(\mathrm{AUC}_{0-\infty}\right) ; \mathrm{K}_{\mathrm{el}}=$ terminal elimination rate constant; $t_{1 / 2}=$ terminal half-life; $T_{\max }=$ time to reach $\mathrm{C}_{\max }$. 
excluding the 24-h sample, were evaluated for their ability to predict the $\mathrm{AUC}_{0-\infty}$ for either formulation. As shown in Table 3, sampling at $3 \mathrm{~h}$ and either $10 \mathrm{~h}$ (formulation A) or $8 \mathrm{~h}$ (formulation B) allows a good estimation of the $\mathrm{AUC}_{0-\infty}$ in each case $\left(\mathrm{R}^{2}=\right.$ 0.93 , bias $<1.2 \%$, precision $=7.3 \%$ ).

\section{Limited-sampling models for $C_{\max }$}

All-subset regression analysis of the plasma concentration data sets (Table 4) revealed that the $\mathrm{C}_{\max }$ for MAA following the administration of either dipyrone formulation can be accurately estimated $\left(R^{2}>0.95\right.$,
Figure 2. A and B, Scatter plots of the relationship between the best-estimated $\mathrm{AUC}_{0-\infty}(\mu \mathrm{g} \mathrm{h}$ $\mathrm{ml}^{-1}$ ) of 4-methylaminoantipyrine (MAA; abscissa) and the $\mathrm{AUC}_{0-\infty}$ derived from the 2-point limitedsampling strategy (LSS) models for each volunteer (ordinate), using the jack-knife approach (Methods). Panel A, Formulation A, data for 5 and 24 h. Panel B, Formulation B, data for 4 and 24 h. C, Scatter plot of the relationship between the best-estimated $A \mathrm{CC}_{0-\infty}\left(\mu \mathrm{g} \mathrm{h} \mathrm{ml} \mathrm{m}^{-1}\right)$ of MAA for dipyrone formulation $A$ (abscissa) and the $\mathrm{AUC}_{0-\infty}$ derived using the 2-point LSS model developed for formulation $B$ and the concentrations observed at the same respective times, but after administration of formulation A (ordinate). The con-

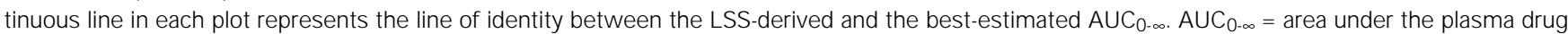
concentration versus time curve from 0 to infinity.

Figure 3. A and B, Scatter plots of the relationship between the best-estimated $C_{\max }(\mu \mathrm{g} / \mathrm{ml})$ of 4 methylaminoantipyrine (MAA; abscissa) and the $\mathrm{C}_{\max }$ derived from the 2-point limited-sampling strategy (LSS) models for each volunteer (ordinate), using the jackknife approach (Methods). Panel A, Formulation $\mathrm{A}$, data for 2 and 3 h. Panel B, Formulation B, data for 1.5 and $2 \mathrm{~h}$. C, Scatter plot of the relationship between the best-estimated $C_{\max }(\mu \mathrm{g} / \mathrm{ml})$ of MAA for dipyrone formulation A (abscissa) and the $\mathrm{C}_{\max }$ derived using the 2-point LSS model developed for formulation $B$ and the concentrations observed at the same respective times, but after administration of formulation $\mathrm{A}$ (ordinate). The continuous line in each plot represents the line of identity between the LSS-derived and the best-estimated $C_{\max }$. $C_{\max }=$ peak plasma concentration.
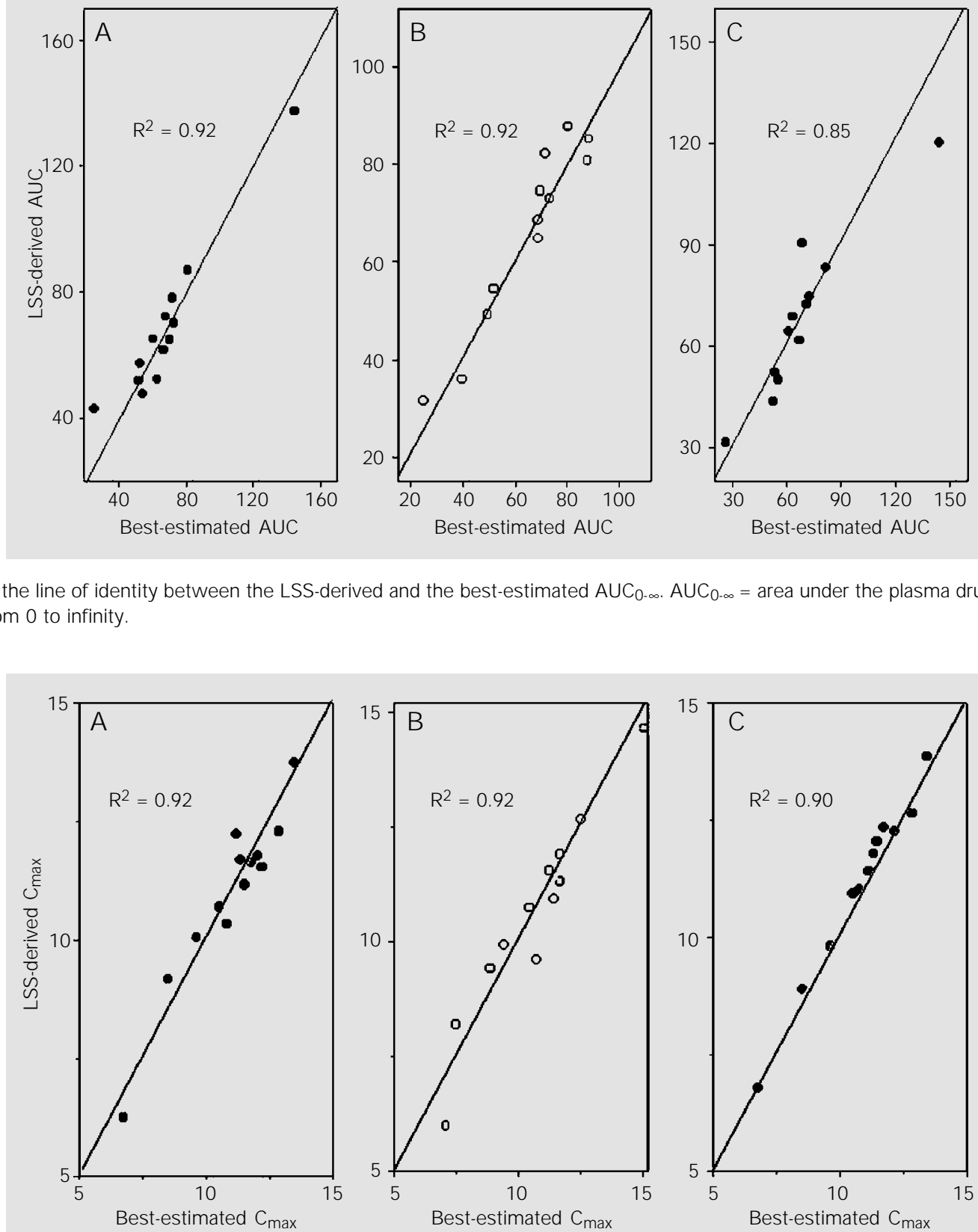
bias $=0.1 \%$, precision $<3.6 \%$ ) by 2 -point LSS equations using data collected at $2 \mathrm{~h}$ and either at $3 \mathrm{~h}$ (formulation $\mathrm{A}$ ) or $1.5 \mathrm{~h}$ (formulation $B$ ). Increasing the number of sampling points to more than two increases $\mathrm{R}^{2}$ by $4 \%$, adds little $(<3 \%)$ to the precision and the bias of the estimates of $\mathrm{C}_{\max }$ of MAA, as compared to the respective values for 2point sampling for each formulation. The jack-knife approach indicated good agreement $\left(R^{2}>0.92\right)$ between the best-estimated $\mathrm{C}_{\max }$ and the 2-point LSS-derived $\mathrm{C}_{\max }$ for either formulation (Figure 3A,B). Figure 3C shows that the most informative 2-point LSS equation developed for formulation $B$ (training set; Methods) predicted well $\left(\mathrm{R}^{2}\right.$ $=0.90)$ the individual $C_{\max }$ values for formulation A.

\section{Limited-sampling models for both $\mathrm{AUC}_{0-\infty}$ and $\mathrm{C}_{\max }$}

Because $\mathrm{AUC}_{0-\infty}$ and $\mathrm{C}_{\max }$ are the standard pharmacokinetic metrics for assessing bioavailability and bioequivalence, it is of practical interest to develop LSS models based on the same sampling times for estimation of these metrics. For this purpose, the plasma MAA concentration data sets for both formulations were pooled and all-subset regression analyses were performed for $\mathrm{AUC}_{0-\infty}$ and $\mathrm{C}_{\max }$. These analyses revealed that a minimum of three sampling points are required for accurate prediction of both pharmacokinetic metrics, the most informative points being 1.5, 4 and $24 \mathrm{~h}$ (Table 5) for estimating both parameters. Diagnostic jackknife plots of the best-estimated versus the LSS-derived $\mathrm{AUC}_{0-\infty}$ or $\mathrm{C}_{\max }$ individual values (Figure 4A,B) showed good agreement $\left(\mathrm{R}^{2}>0.90\right.$, bias $<0.8 \%$, precision between 3.9 and $7.7 \%$ ) between observed and predicted quantities.

\section{Validation of the LSS models using simulated data}

The best-estimated $\mathrm{AUC}_{0-\infty}$ and $\mathrm{C}_{\max }$ for
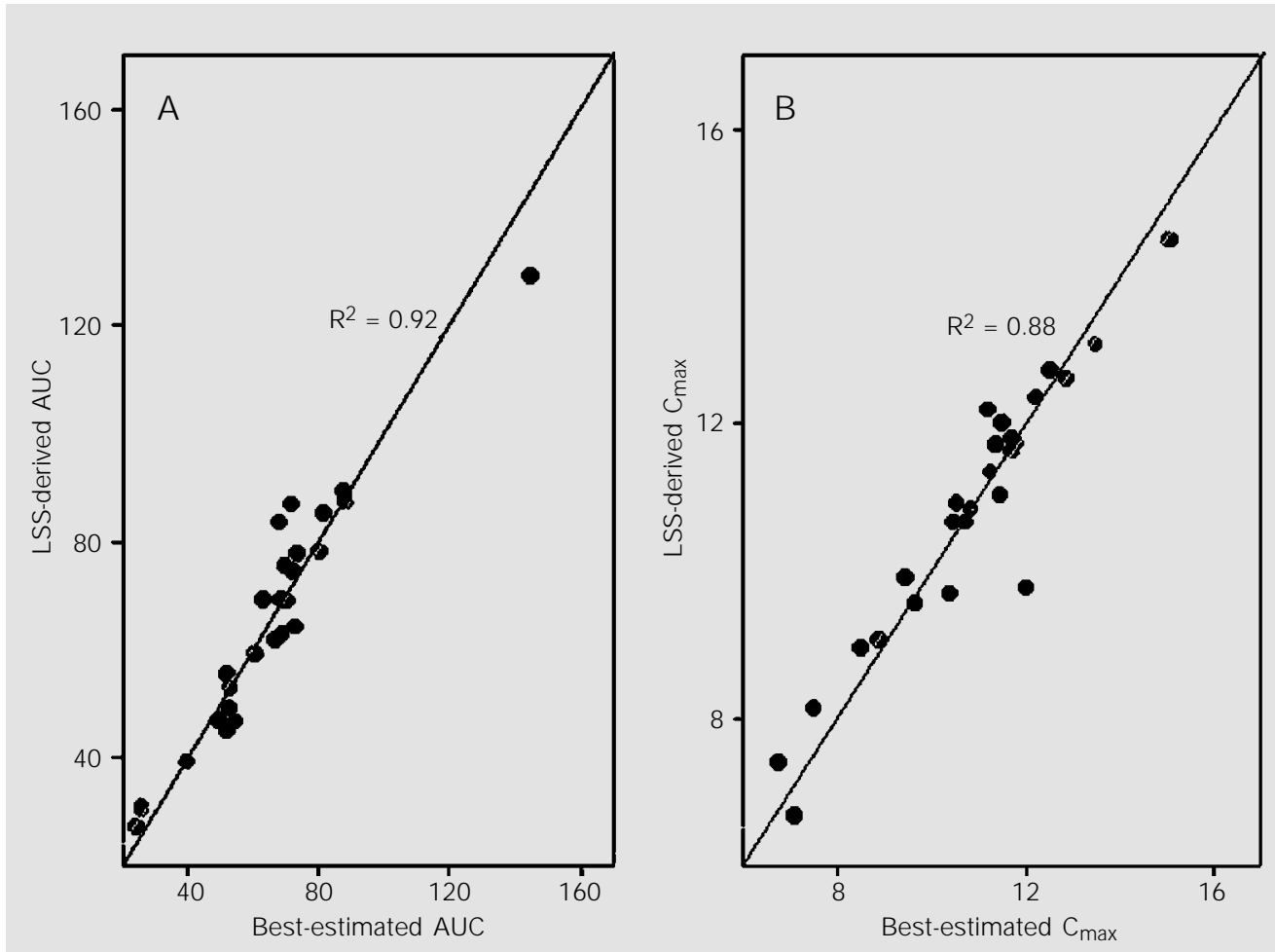

Figure 4. A, B, Scatter plots of the relationship between the best-estimated $\mathrm{AUC}_{0-\infty}(\mu \mathrm{g} \mathrm{h}$ $\left.\mathrm{ml}^{-1}\right)$ or $C_{\max }(\mu \mathrm{g} / \mathrm{ml})$ of MAA (abscissa) and the corresponding parameter values derived from the 3-point limited-sampling strategy (LSS) models (see equations in Table 5) for each volunteer (ordinate), using the jack-knife approach (Methods). The continuous line in each plot represents the line of identity between the LSS-derived and the best-estimated $\mathrm{AUC}_{0-\infty}$ (A) or $\mathrm{C}_{\max }(\mathrm{B}) . \mathrm{AUC}_{0-\infty}=$ area under the plasma drug concentration versus time curve from 0 to infinity; $C_{\max }=$ peak plasma concentration. 
the 24 simulated data sets (Methods) were $56.2 \pm 19.5($ mean $\pm \mathrm{SD})$ and $8.45 \pm 1.52$, respectively. The corresponding values for the LSS-estimated metrics using the 3point LSS equations shown in Table 5 were $54.8 \pm 18.3$ and $9.18 \pm 0.86$. The best-estimated and the LSS-derived metrics were closely correlated: $\mathrm{R}^{2}=0.98$ and 0.88 , bias $=-1.93 \%$ and $8.54 \%$, precision $=8.65 \%$ and $5.16 \%$, for $\mathrm{AUC}_{0-\infty}$ and $\mathrm{C}_{\max }$, respectively.

\section{Bioequivalence analysis}

The $90 \%$ confidence intervals of the individual percent ratios (formulation $\mathrm{A} /$ formulation $\mathrm{B}$ ) of the $\log$-transformed $\mathrm{C}_{\max }$ and $\mathrm{AUC}_{0-\infty}$ of MAA, calculated for the best-estimated and for the LSS-derived metrics, were closely similar and within the accepted bioequivalence range of $80-125 \%$ (Table 6). The power of ANOVA was also comparable for the bestestimated and the LSS-generated data sets.

Table 2. $R^{2}$, bias (MD\%) and precision (MAD\%) of the best linear equations for $n$ sample times derived using the all-subset regression approach to estimate the $\mathrm{AUC}_{0-\infty}$ for each subject for formulations $\mathrm{A}$ and $\mathrm{B}$.

\begin{tabular}{|c|c|c|c|c|c|c|}
\hline $\mathrm{N}$ & Sample times (h) & $R^{2}$ & MD\% & $\pm \mathrm{SD}$ & MAD\% & $\pm \mathrm{SD}$ \\
\hline \multicolumn{7}{|c|}{ Formulation A } \\
\hline 1 & 10 & 0.887 & -1.79 & 16.55 & 11.41 & 11.61 \\
\hline 2 & 5,24 & 0.960 & -1.46 & 11.96 & 8.30 & 8.40 \\
\hline 3 & $3,10,24$ & 0.981 & -0.73 & 7.92 & 5.02 & 6.00 \\
\hline 4 & $0.33,5,10,24$ & 0.991 & -0.53 & 4.77 & 3.60 & 3.01 \\
\hline 5 & $0.33,2,4,10,24$ & 0.996 & -0.31 & 3.51 & 2.66 & 2.18 \\
\hline 6 & $0.33,0.66,2,3,10,24$ & 0.997 & -0.20 & 2.47 & 1.92 & 1.47 \\
\hline 7 & $0.33,0.66,2,3,5,10,24$ & 0.997 & -0.23 & 2.46 & 1.91 & 1.47 \\
\hline 8 & $0.33,0.66,1.5,3,4,5,8,10$ & 0.999 & -0.14 & 1.42 & 1.09 & 0.87 \\
\hline 9 & $0.33,0.66,1,1.5,3,4,5,8,10$ & 0.999 & -0.04 & 0.89 & 0.63 & 0.61 \\
\hline 10 & $0.33,0.66,1,1.5,2,3,4,5,8,10$ & 0.999 & -0.07 & 0.93 & 0.63 & 0.67 \\
\hline \multicolumn{7}{|c|}{ Formulation B } \\
\hline 1 & 8 & 0.885 & -1.92 & 12.50 & 8.95 & 8.68 \\
\hline 2 & 4,24 & 0.964 & -0.67 & 7.20 & 5.38 & 4.58 \\
\hline 3 & $0.33,4,24$ & 0.981 & -0.42 & 4.87 & 3.82 & 2.85 \\
\hline 4 & $0.33,4,10,24$ & 0.995 & -0.18 & 2.33 & 1.69 & 1.54 \\
\hline 5 & $0.33,1.5,4,10,24$ & 0.997 & $-7.04^{-02}$ & 1.71 & 1.38 & 0.94 \\
\hline 6 & $0.33,0.66,1,4,10,24$ & 0.998 & $6.75^{-02}$ & 1.57 & 1.22 & 0.92 \\
\hline 7 & $0.33,0.66,1,3,4,10,24$ & 0.998 & $8.39^{-02}$ & 1.62 & 1.12 & 1.13 \\
\hline 8 & $0.33,0.66,1,3,4,8,10,24$ & 0.998 & $8.67^{-02}$ & 1.65 & 1.16 & 1.14 \\
\hline 9 & $0.33,0.66,1,1.5,3,4,8,10,24$ & 0.998 & $9.15^{-02}$ & 1.63 & 1.11 & 1.15 \\
\hline 10 & $0.33,0.66,1,1.5,2,3,4,8,10,24$ & 0.998 & $8.99^{-02}$ & 1.62 & 1.11 & 1.14 \\
\hline
\end{tabular}

$\mathrm{MD} \%$ and $\mathrm{MAD} \%=$ mean and mean absolute percentage of difference, respectively. $\mathrm{AUC}_{0-\infty}=$ area under the plasma drug concentration versus time curve from 0 to infinity.

Table 3. $R^{2}$, bias (MD\%) and precision (MAD\%) of the best linear equations for two sample times, excluding sampling at $>10 \mathrm{~h}$, derived using the all-subset regression approach to estimate the $\mathrm{AUC}_{0-\infty}$ for each subject for formulations $\mathrm{A}$ and $\mathrm{B}$.

\begin{tabular}{lcccrrr}
\hline Formulation & LSS equation & $\mathrm{R}^{2}$ & $\mathrm{MD} \%$ & $\pm \mathrm{SD}$ & $\mathrm{MAD} \%$ & $\pm \mathrm{SD}$ \\
\hline $\mathrm{A}$ & $3.39+3.27 * \mathrm{C}_{3}+23.04^{*} \mathrm{C}_{10}$ & 0.939 & -0.04 & 10.20 & 7.27 & 6.85 \\
B & $8.48+3.65 * \mathrm{C}_{3}+10.54^{*} \mathrm{C}_{8}$ & 0.928 & -1.18 & 9.25 & 6.85 & 6.02
\end{tabular}

$\mathrm{MD} \%$ and $\mathrm{MAD} \%=$ mean and mean absolute percentage of difference, respectively. $\mathrm{AUC}_{0_{-\infty}}=$ area under the plasma drug concentration versus time curve from 0 to infinity. LSS = limited-sampling strategy. 
Table 4. $\mathrm{R}^{2}$, bias (MD\%) and precision (MAD\%) of the best linear equations for $n$ sample times derived using the all-subset regression approach to estimate the $C_{\max }$ for each subject for formulations $A$ and $B$.

\begin{tabular}{|c|c|c|c|c|c|c|}
\hline $\mathrm{N}$ & Sample times (h) & $\mathrm{R}^{2}$ & MD\% & $\pm \mathrm{SD}$ & MAD\% & $\pm \mathrm{SD}$ \\
\hline \multicolumn{7}{|l|}{ Formulation A } \\
\hline 1 & 2 & 0.837 & -0.43 & 6.74 & 4.95 & 4.36 \\
\hline 2 & 2,3 & 0.954 & -0.10 & 3.63 & 3.07 & 1.72 \\
\hline 3 & $1,2,3$ & 0.976 & $-2.66^{-02}$ & 2.93 & 2.36 & 1.60 \\
\hline 4 & $1,1.5,2,3$ & 0.978 & $-2.23^{-02}$ & 2.81 & 2.16 & 1.69 \\
\hline 5 & $1,2,3,5,10$ & 0.980 & $-6.22^{-03}$ & 2.61 & 2.01 & 1.55 \\
\hline 6 & $1,1.5,2,4,10,24$ & 0.985 & $-3.98^{-03}$ & 2.11 & 1.63 & 1.25 \\
\hline 7 & $1,1.5,2,4,8,10,24$ & 0.986 & $6.12^{-03}$ & 2.12 & 1.72 & 1.14 \\
\hline 8 & $1,1.5,2,3,4,8,10,24$ & 0.987 & $8.40^{-03}$ & 2.01 & 1.60 & 1.14 \\
\hline 9 & $1,1.5,2,3,4,5,8,10,24$ & 0.987 & $1.17^{-02}$ & 2.02 & 1.58 & 1.18 \\
\hline 10 & $0.33,0.66,1.5,2,3,4,5,8,10,24$ & 0.999 & $1.50^{-03}$ & 0.52 & 0.26 & 0.44 \\
\hline \multicolumn{7}{|l|}{ Formulation B } \\
\hline 1 & 1.5 & 0.936 & -0.19 & 5.80 & 4.68 & 3.15 \\
\hline 2 & $1.5,2$ & 0.965 & -0.10 & 4.42 & 3.63 & 2.30 \\
\hline 3 & $1.5,2,24$ & 0.971 & $-9.03-02$ & 3.85 & 2.97 & 2.30 \\
\hline 4 & $1.5,8,10,24$ & 0.985 & $-8.05^{-03}$ & 2.73 & 2.29 & 1.34 \\
\hline 5 & $1.5,4,8,10,24$ & 0.989 & $-1.47^{-02}$ & 2.44 & 1.76 & 1.61 \\
\hline 6 & $0.66,1,1.5,3,4,10$ & 0.990 & $-4.77-02$ & 2.04 & 1.55 & 1.24 \\
\hline 7 & $0.66,1,1.5,2,4,5,24$ & 0.998 & $-7.94^{-03}$ & 0.86 & 0.74 & 0.40 \\
\hline 8 & $0.66,1,1.5,2,4,5,10,24$ & 0.999 & $8.38^{-03}$ & 0.59 & 0.44 & 0.37 \\
\hline 9 & $0.66,1,1.5,2,4,5,8,10,24$ & 0.999 & $3.25^{-04}$ & 0.22 & 0.16 & 0.14 \\
\hline 10 & $0.66,1,1.5,2,3,4,5,8,10,24$ & 0.999 & $7.27^{-04}$ & 0.22 & 0.16 & 0.13 \\
\hline \multicolumn{7}{|c|}{ The most informative 2-point equation for each formulation. } \\
\hline & LSS equation & $R^{2}$ & MD\% & $\pm \mathrm{SD}$ & MAD\% & $\pm \mathrm{SD}$ \\
\hline Formulation A & $2.05+0.52 * C_{2}+0.42 * C_{3}$ & 0.954 & -0.10 & 3.63 & 3.07 & 1.72 \\
\hline Formulation B & $0.66+0.66 * C_{1.5}+0.34 * C_{2}$ & 0.965 & -0.10 & 4.42 & 3.63 & 2.30 \\
\hline
\end{tabular}

$M D \%$ and $M A D \%=$ mean and mean absolute percentage of difference, respectively. $C_{\max }=$ peak plasma concentration. LSS = limited-sampling strategy.

Table 5. $\mathrm{R}^{2}$, bias (MD\%) and precision (MAD\%) of the best linear equations for the same three sample times, derived using the all-subset regression approach to estimate both the $A U C_{0-\infty}$ and $C_{\max }$ for each subject for formulations $\mathrm{A}$ and $\mathrm{B}$.

\begin{tabular}{|c|c|c|c|c|c|c|}
\hline Parameter & LSS equation & $R^{2}$ & MD\% & $\pm \mathrm{SD}$ & MAD\% & $\pm \mathrm{SD}$ \\
\hline & $.29 * C_{1.5}$ & 0.9 & -0.65 & 9.60 & 7.4 & 5.97 \\
\hline $\mathrm{C}_{\max }$ & $1.86+0.72 * C_{1.5}+0.26 * C_{4}-0.40 * C_{24}$ & 0.890 & -0.37 & 6.19 & 4.3 & 4.4 \\
\hline
\end{tabular}

$\mathrm{AUC}_{0-\infty}=$ area under the plasma drug concentration versus time curve from 0 to infinity. For other abbreviations, see legend to Table 4.

Table 6. Bioequivalence assessment of formulations A and B using the best-estimated and the limitedsampling strategy (LSS)-derived data.

\begin{tabular}{lcr}
\hline Data source & AUC $_{0-\infty}{ }^{a}$ & $C_{\max ^{a}}$ \\
\hline Best-estimated & $101.8(94.5-109.7), 0.99$ & $100.2(94.2-106.5), 0.99$ \\
LSS-derived & $103.2(98.2-108.4), 0.99$ & $97.9(93.8-102.2), 0.99$ \\
\hline
\end{tabular}

andividual ratios. Data are reported as geometric mean $\left(90 \%\right.$ confidence interval), power. $\mathrm{AUC}_{0_{-\infty}}=$ area under the plasma drug concentration versus time curve from 0 to infinity; $C_{\max }=$ peak plasma concentration. 


\section{Discussion}

This paper describes for the first time the development of LSS for predicting both the plasma $\mathrm{AUC}_{0-\infty}$ and $\mathrm{C}_{\max }$ of MAA, the main active metabolite of the prodrug dipyrone. These strategies were developed using data from a bioequivalence study in which a relatively large number of plasma samples $(\mathrm{N}=$ 336) were collected from closely monitored healthy volunteers. Our LSS analysis and validation procedures show that the $\mathrm{AUC}_{0-\infty}$ and $\mathrm{C}_{\max }$ of MAA following oral administration of single $600 \mathrm{mg}$ doses of dipyrone can be determined accurately using only two plasma samples. Choosing three or more samples adds little to the accuracy and precision of the estimates (Tables 2 and 4). The statistical principle of parsimony advises in favor of models with fewer parameters and therefore we settled for 2-sample regressions for independent estimation of MAA $\mathrm{AUC}_{0-\infty}$ or $\mathrm{C}_{\max }$. Validation tests, namely the jack-knife prediction and the use of training sets, provided strong support for the conclusion that both the $\mathrm{AUC}_{0-\infty}$ and the $\mathrm{C}_{\max }$ of MAA following administration of single doses of dipyrone can be accurately estimated by the 2-point LSS models developed in this study. The fact that the interindividual variability in several pharmacokinetic parameters was relatively large heightens the methodological and practical value of the proposed LSS models.

Although the most informative 2-point regression equations for predicting the $\mathrm{AUC}_{0-\infty}$ require sampling at $24 \mathrm{~h}$, when the MAA plasma concentrations are close to the quantification limit of the analytical method used, we showed that accurate estimates of $\mathrm{AUC}_{0-\infty}$ can also be obtained using 2-point LSS models, which exclude the 24-h sample. Other criteria that determine the practical value of LSS models include avoiding sampling at "unsocial" hours or long after drug administration (to reduce the duration of the study) and to use the same sampling times but different regression coefficients to obtain the pharmacokinetic metrics of interest. In the case of bioequivalence studies, these metrics are $\mathrm{AUC}_{0-\infty}$ and $\mathrm{C}_{\max }$, and the present study reveals that 3-point LSS models based on sampling at 1.5, 4 and $24 \mathrm{~h}$ provide the information required for accurate estimation of both of these metrics for MAA following the administration of dipyrone. The corresponding LSS equations were validated in the same group of volunteers using the jackknife approach, and also in a simulated group of 24 "subjects" generated by a pharmacokinetic model applied to concentration-inplasma data from a previously published study in which the reference dipyrone formulation (Novalgin ${ }^{\circledR}$ ) was used (6). The latter observation suggests to us that the applicability of the LSS models developed here is not limited to the formulations and population studied. Nevertheless, we are aware of the potential limitations of these LSS models when applied to other dipyrone formulations, which could change the absorption profile of the drug, and consequently the pharmacokinetic parameters being analyzed.

Finally, the $\mathrm{AUC}_{0-\infty}$ and $\mathrm{C}_{\max }$ of MAA derived by the 3-point LSS models developed in the present study allowed precise assessment of the bioequivalence between the two dipyrone formulations tested. This observation adds support to previous proposals $(7,8,16)$ that LSS models are valuable tools for bioequivalence trials, with the advantage of reducing the costs of sampling and analysis, as well as the time required for completion of the trial. 


\section{References}

1. Levy M, Zylber-Katz E \& Rosenkranz B (1995). Clinical phamacokinetics of dipyrone and its metabolites. Clinical Pharmacokinetics, 28: 216-234.

2. Volz M \& Kellner H-M (1980). Kinetics and metabolism of pyrazolones (propylphenazone, aminopyrine and dipyrone). British J ournal of Clinical Pharmacology, 10: 299S-308S.

3. Igushi S, Goromaru T \& Noda A (1975). A new metabolite of aminopyrine (aminophenazone) in man, 4-formyl-aminoantipyrine. Chemical and Pharmaceutical Bulletin, 23: 932-934.

4. Levy M, Flusser D, Zylber-Katz E \& Granit $L$ (1984). Plasma kinetics of dipyrone metabolites in rapid and slow acetylators. European J oumal of Clinical Pharmacology, 27: 453-458.

5. Ribeiro FM, Estrela RCE \& Suarez-Kurtz G (2000). Limited-sampling models for 4methylaminoantipyrine - a dipyrone metabolite - based on data from a bioequivalence study. XVI Latin American Congress of Pharmacology, Águas de Lindóia, SP, Brazil. Abstract 234.
6. Vlahov V, Badian M, Verho M \& Bacracheva N (1990). Pharmacokinetics of metamizol metabolites in healthy subjects after a single oral dose of metamizol sodium. European J oumal of Clinical Pharmacology, 38: 61-65.

7. Suarez-Kurtz G, Bozza FA, Vicente FL, Ponte CG \& Struchiner CJ (1999). Limited-sampling strategy models for itraconazole and hydroxy-itraconazole based on data from a bioequivalence study. Antimicrobial Agents and Chemotherapy, 43: 134-140.

8. Suarez-Kurtz G, Ponte CG, Buy VLM \& Struchiner CJ (1999). Limited-sampling strategy models for estimating the area under the plasma concentration-time curve for amlodipine. European J ournal of Clinical Pharmacology, 55: 651-657.

9. WinNonlin Pro 3.1 (2000). Pharsight Corporation, Cary, NC, USA.

10. J odrell DI, Murray LS, Hawtof J , Graham MA \& Egorin MJ (1996). A comparison of methods for LSS design using data from a phase I trial of the anthapyrazole DuP941. Cancer Chemotherapy and Pharma- cology, 37: 356-362.

11. Furnival GM \& Wilson J r RW (1974). Regressions by leaps and bounds. Technometrics, 16: 499-511.

12. Splus 4 (1997). Guide to Statistics. Data Analysis Products Division, Mathsoft, Seattle, WA, USA.

13. Ingram $D \&$ Block $R$ (1984). Mathematical Methods in Medicine. Part 1. Statistical and Analytical Techniques. J ohn Wiley and Sons, Chichester, England.

14. D'Argenio DZ \& Schumizky A (1997). ADAPT II User's Guide: Pharmacokinetic/ Pharmacodynamic Systems Analysis Software. Biomedical Simulations Resource, Los Angeles, CA, USA.

15. Schuimann DJ (1987). A comparison of the two one-sided tests procedure and the power approach for assessing the equivalence of average bioavailability. $J$ oumal of Pharmacokinetics and Biopharmaceutics, 15: 657-680.

16. Mahmood I, Chamberlin N \& Tammara V (1997). A limited sampling approach in bioequivalence studies. Therapeutic Drug Monitoring, 19: 413-419. 